\title{
Strain elastography of palatal tumors in conjunction with intraoral ultrasonography, computed tomography, and magnetic resonance imaging: 2 case reports
}

\author{
Ichiro Ogura $\mathbb{D}^{1, *}$, Hiroo Toshima $\mathbb{D}^{1}$, Tohru Akashiba $\mathbb{D}^{2}$, Junya Ono $\mathbb{D}^{3}$, Yasuo Okada $\mathbb{D}^{3}$ \\ ${ }^{1}$ Department of Oral and Maxillofacial Radiology, The Nippon Dental University School of Life Dentistry at Niigata, Niigata, Japan \\ ${ }^{2}$ Department of Oral and Maxillofacial Surgery, The Nippon Dental University Niigata Hospital, Niigata, Japan \\ ${ }^{3}$ Department of Pathology, The Nippon Dental University School of Life Dentistry at Niigata, Niigata, Japan
}

\section{ABSTRACT}

Computed tomography (CT) and magnetic resonance imaging (MRI) can be useful for the evaluation of palatal lesions, and strain elastography (performed together with intraoral ultrasonography) is a relatively new sonographic imaging modality. This report describes 2 clinical cases in which strain elastography was used to assess palatal tumors in conjunction with intraoral ultrasonography, CT, and MRI. In the first case, diagnosed as a myoepithelioma, the strain was determined to be $0.000 \%$ (strain of normal tissue, $0.556 \%$ ). In the second case, diagnosed as an adenoid cystic carcinoma, the determined strain was $0.000 \%$ (strain of normal tissue, $1.077 \%$ ). Therefore, we conclude that intraoral strain elastography can be useful for evaluating palatal lesions.(Imaging Sci Dent 2020; 50: 73-9)

KEY WORDS: Elasticity Imaging Techniques; Ultrasonography; Palatal Neoplasms

Ultrasonography is most extensively used for preoperative imaging as part of the diagnosis of oral and maxillofacial lesions. ${ }^{1}$ In recent years, shear wave elastography has been shown to be an effective technique for the objective and quantitative diagnosis of cervical lymph node metastases of oral carcinoma. ${ }^{2,3}$

Intraoral ultrasonography has been used to assess features of tongue cancer, including the border, size, location, depth, vascularity, and histological findings of the mass. ${ }^{4,5}$ Strain elastography (performed together with intraoral ultrasonography) is relatively new to sonographic imaging, and the use of intraoral strain elastography for tongue cancer has been recently reported in the literature. ${ }^{6,7}$

Computed tomography $(\mathrm{CT}),{ }^{8,9}$ magnetic resonance imaging (MRI) ${ }^{10}$ and ultrasonography ${ }^{11}$ can be useful for the preoperative evaluation of palatal lesions. However, to the best of our knowledge, intraoral strain elastography of pal-

This work was supported by JSPS KAKENHI Grant Number JP 18K09754.

Received September 24, 2019; Revised October 7, 2019; Accepted October 14, 2019 *Correspondence to : Prof. Ichiro Ogura

Department of Oral and Maxillofacial Radiology, The Nippon Dental University School of Life Dentistry at Niigata, 1-8 Hamaura-cho, Chuo-ku, Niigata, Niigata 951-8580, Japan

Tel) 81-25-267-1500,E-mail) ogura@ngt.ndu.ac.jp atal lesions has not been extensively described in the literature. This report describes 2 clinical cases in which strain elastography was used to assess palatal tumors in conjunction with intraoral ultrasonography, CT, and MRI.

\section{Case Report}

\section{Case 1}

A 54-year-old woman was referred to our hospital with a chief concern of swelling on the right palate that had developed over 6 months. The patient underwent intraoral strain elastography with a linear 17-MHz transducer (Aplio 300; Canon Medical Systems, Otawara, Japan), CT with a 16-detector CT scanner (Aquilion TSX-101A; Canon Medical Systems, Otawara, Japan), and MRI with a 1.5-T magnetic resonance unit(EXCELART Vantage MRT-2003; Canon Medical Systems) at our university hospital in accordance with the institutional protocol. ${ }^{7,12}$

On ultrasonography, the tumor showed a clear boundary, hypoechoic echogenicity, homogeneous internal architecture, and no vascular signals (Figs. 1A and B). The strain values of the normal tissue and the tumor were $0.556 \%$ and $0.000 \%$, respectively (Figs. 1C and D). CT showed a 

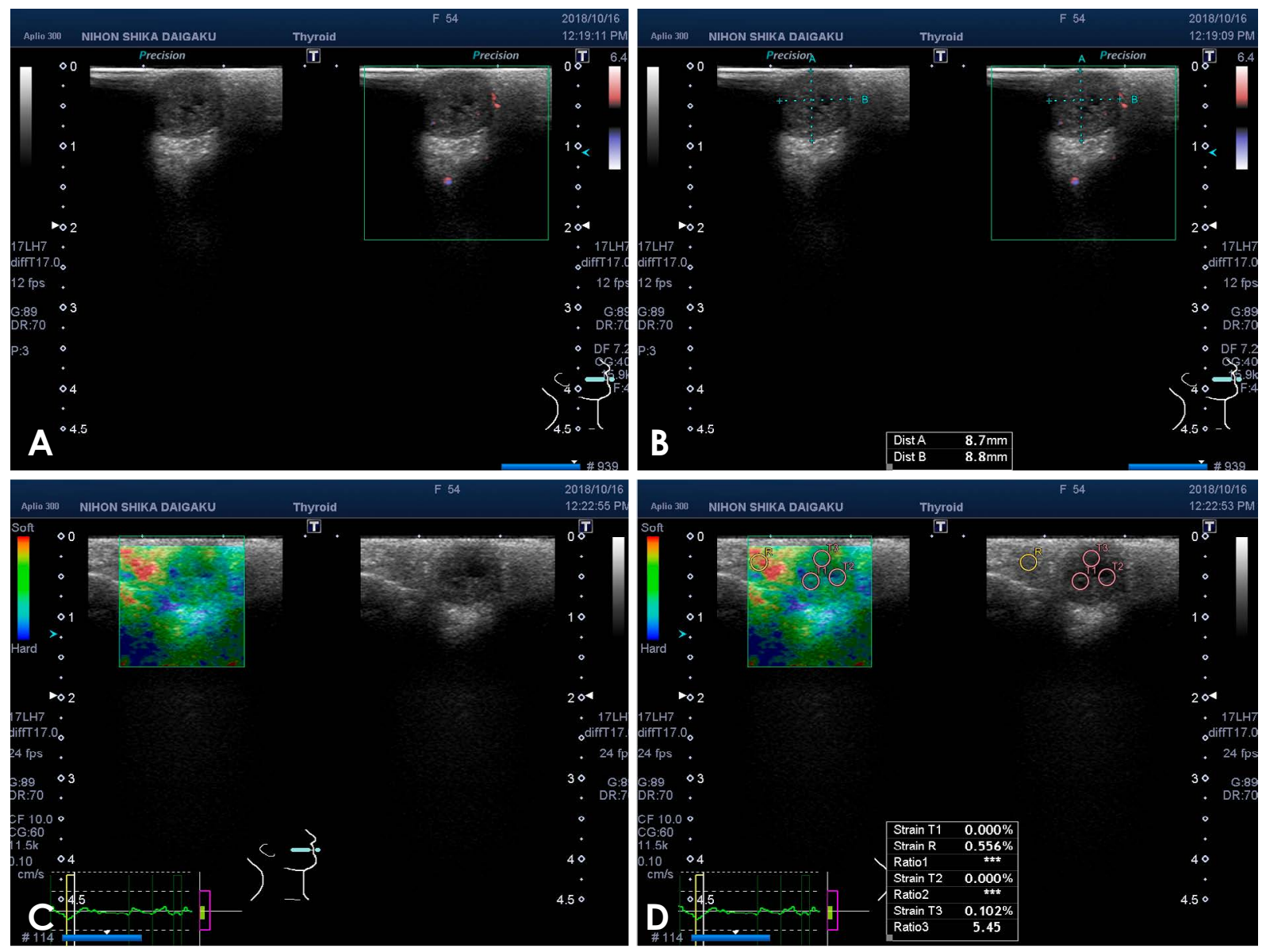

Fig. 1. Ultrasonography of a myoepithelioma of the palate in a 54-year-old woman. A and B. Color Doppler ultrasonography. C. Strain elastography. D. The strain values of the normal tissue and tumor in the palate were R and T1-3, respectively.
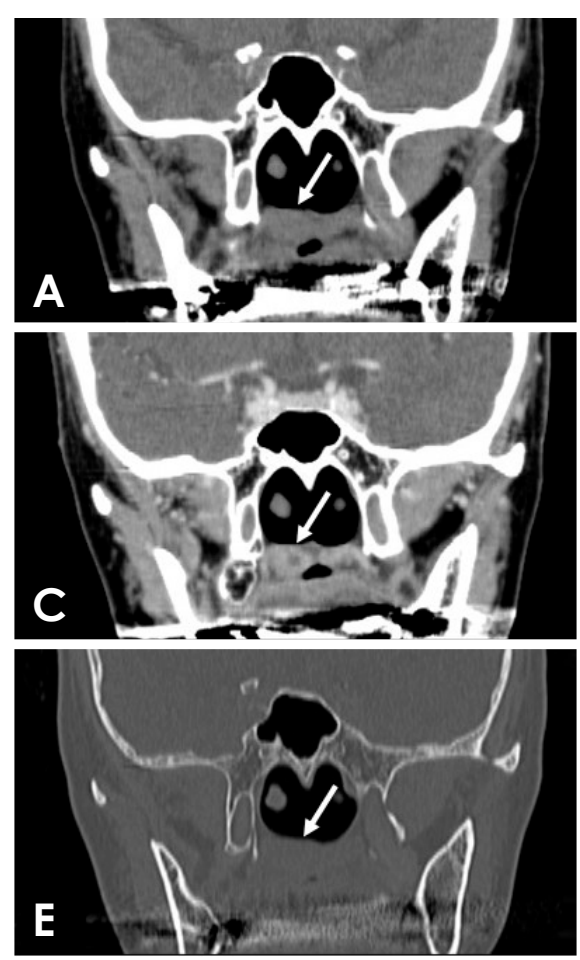
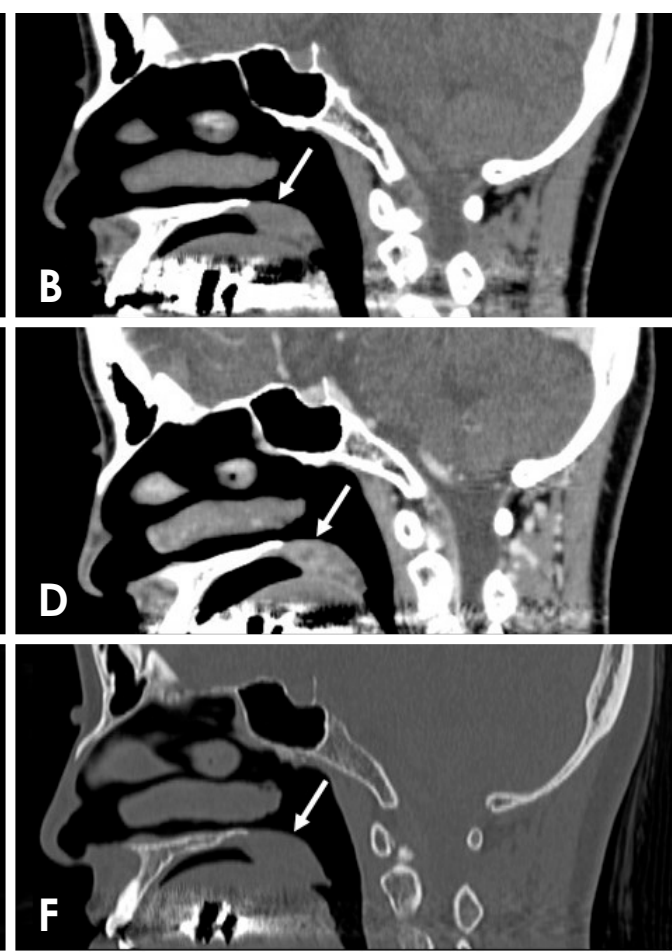

Fig. 2. Computed tomography (CT) of a myoepithelioma of the palate in a 54-year-old woman (arrows). A. Coronal soft-tissue algorithm CT image. B. Sagittal soft-tissue algorithm CT image. C. Coronal contrast-enhanced CT image. D. Sagittal contrast-enhanced CT image. E. Coronal bone-tissue algorithm CT image. F. Sagittal bone-tissue algorithm CT image. 

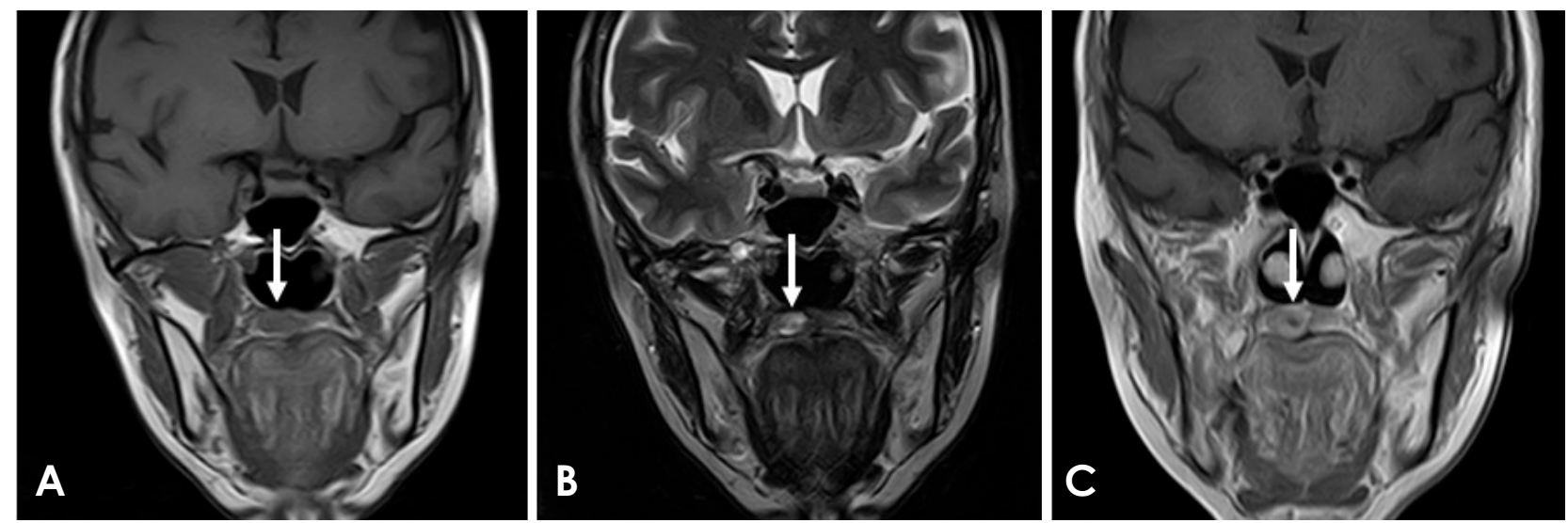

Fig. 3. Magnetic resonance image of a myoepithelioma of the palate in a 54-year-old woman (arrows). A. Coronal T1-weighted image. B. Coronal T2-weighted image. C. Coronal post-contrast T1-weighted image.
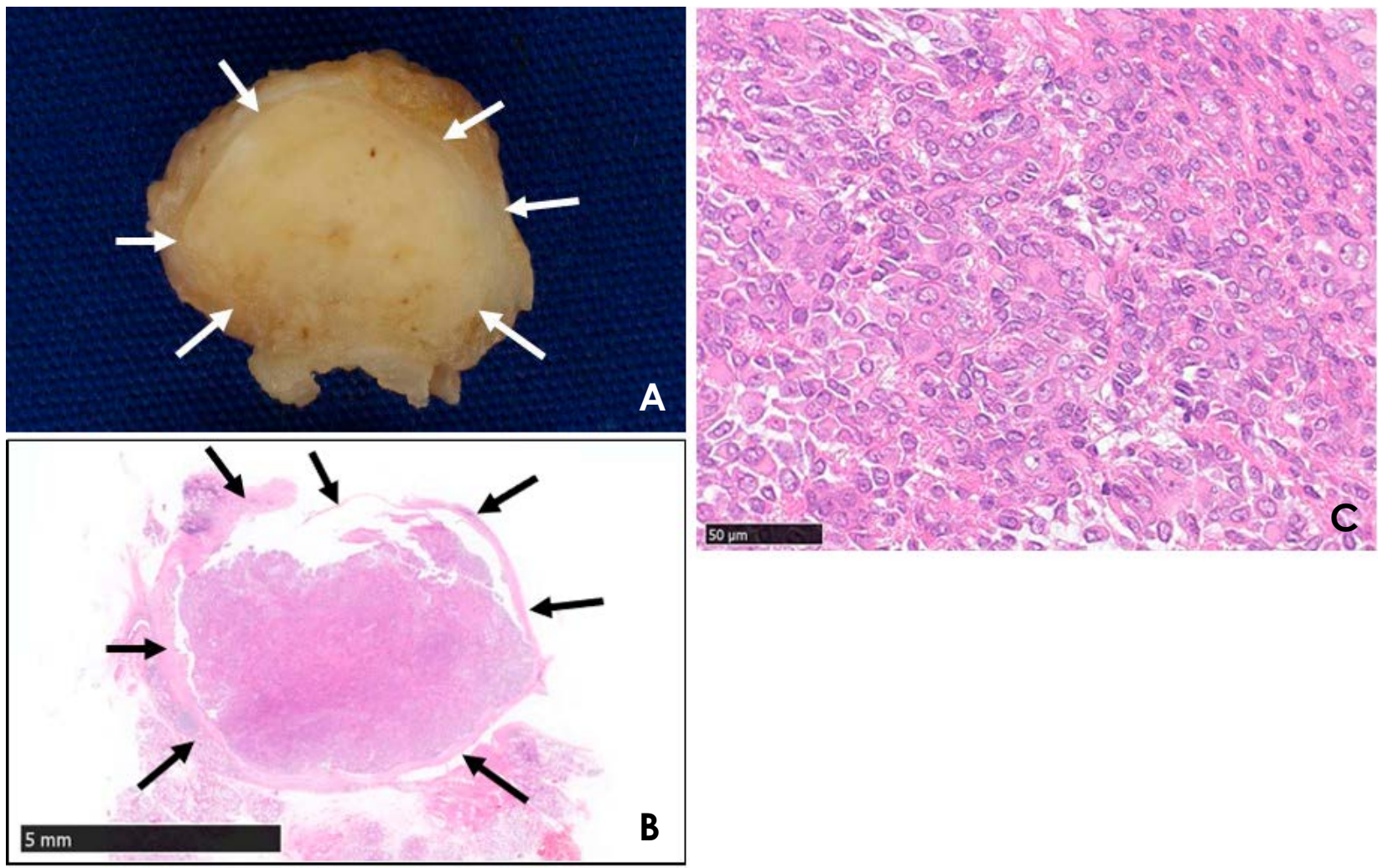

Fig. 4. Specimen and histopathological exam of a myoepithelioma of the palate in a 54-year-old woman. On the cut surface of the tumor (A) and on the low-magnification view (B) of the surgical specimen (hematoxylin and eosin [H\&E] stain $\times 1$ magnification; scale bar, 5 mm), a thin, discrete fibrous capsule was visible (arrows). C. Epithelioid, spindle, and plasmacytoid neoplastic myoepithelial cells were observed (H\&E stain $\times 40$ magnification; scale bar, $50 \mu \mathrm{m})$.

mass (Figs. 2A and B), and contrast-enhanced CT showed an enhancing mass (Figs. 2C and D). Coronal and sagittal bone-tissue algorithm CT showed no osteolytic lesion, but did not show perineural spread (Figs. 2E and F). On MRI, a coronal T1-weighted image revealed homoge- neous low-signal intensity (Fig. 3A), a T2-weighted image showed heterogeneous high-signal intensity (Fig. 3B), and a post-contrast T1-weighted image showed an enhancing mass (Fig. 3C).

Regarding the cut surface of the tumor (Fig. 4A) and the 

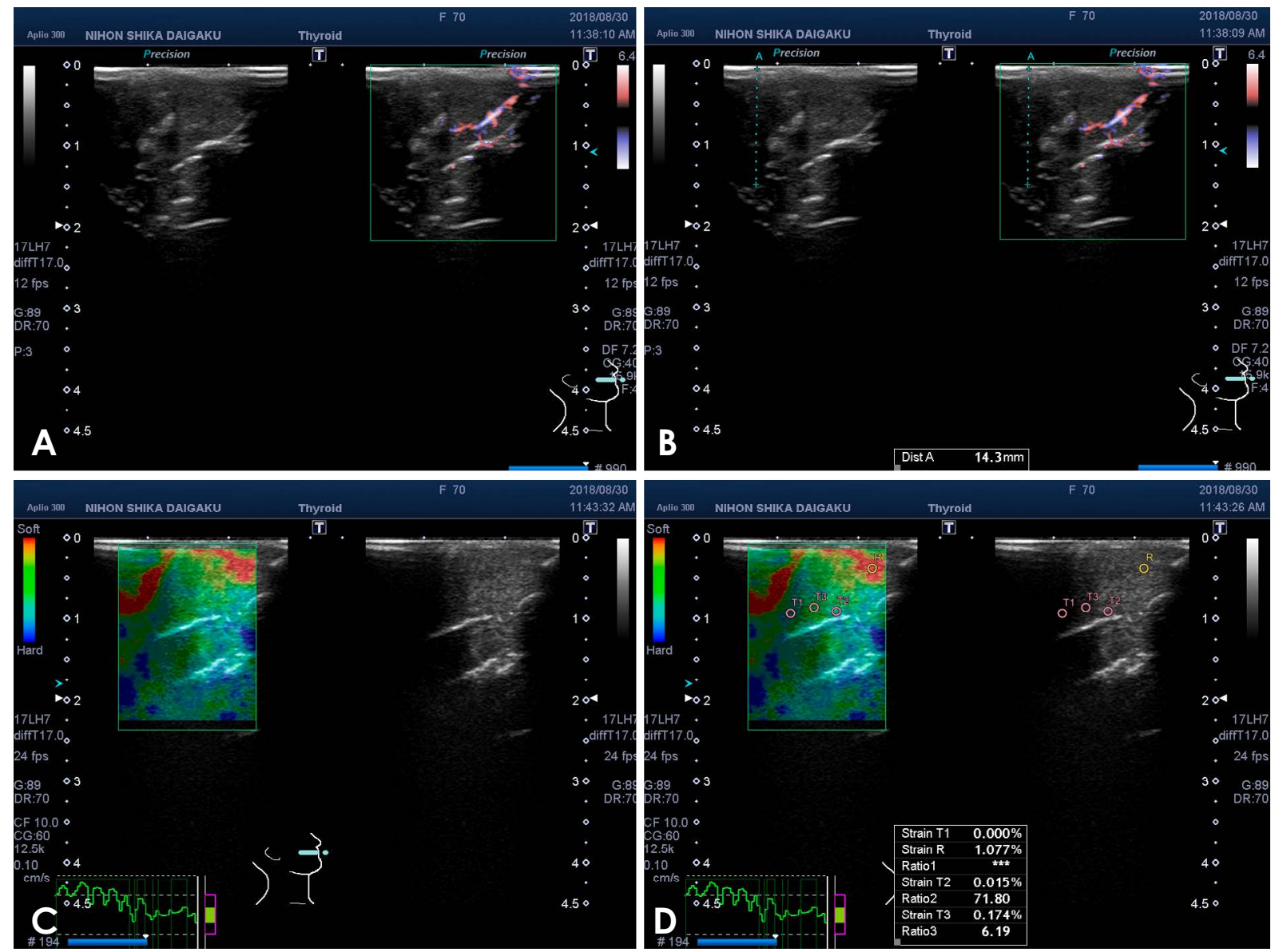

Fig. 5. Ultrasonography of an adenoid cystic carcinoma of the palate in a 70-year-old woman. A and B. Color Doppler ultrasonography. C. Strain elastography. D. The strain values of the normal tissue and tumor in the palate were R and T1-3, respectively.

low-magnification view (Fig. 4B; $\times 1$ magnification; scale bar, $5 \mathrm{~mm}$ ) of the surgical specimen, a thin, discrete fibrous capsule was observed. Furthermore, epithelioid, spindle, and plasmacytoid-neoplastic myoepithelial cells were observed (Fig. 4C; $\times 40$ magnification; scale bar, $50 \mu \mathrm{m}$ ). The histopathological diagnosis was myoepithelioma.

\section{Case 2}

A 70-year-old woman was referred to our hospital with a chief concern of swelling on the right palate that had developed over 3 months. The patient underwent ultrasonography, CT, and MRI at our university hospital in accordance with the institutional protocol.

On ultrasonography, the tumor showed an unclear boundary, isoechoic echogenicity, homogeneous internal architecture, and vascular signals (Figs. 5A and B). The strain values of the normal tissue and the tumor were $1.077 \%$ and $0.000 \%$, respectively (Figs. 5C and D). CT showed a mass (Figs. 6A and B), and contrast-enhanced CT showed an enhancing mass (Figs. 6C and D). Coronal and sagittal bone-tissue algorithm CT images showed an osteolytic lesion and perineural spread (Figs. 6E and F). On MRI, a coronal T1-weighted image revealed homogeneous low-signal intensity (Fig. 7A), a T2-weighted image showed heterogeneous high-signal intensity (Fig. 7B), and a post-contrast T1-weighted image showed an enhancing mass (Fig. 7C).

Regarding the coronal segment of the right maxillary first molar (Fig. 8A, real view) and the low-magnification view (Fig. $8 \mathrm{~B} ; \times 1$ magnification; scale bar, $5 \mathrm{~mm}$ ) of the surgical specimen, the tumor showed aggressive bone destruction and extension into the maxillary sinus $(*)$ and nasal cavity $(\star)$. Furthermore, cribriform and tubular patterns were observed (Fig. 8C; $\times 40$ magnification; scale bar, $50 \mu \mathrm{m})$. The histopathological diagnosis was adenoid cystic carcinoma.

\section{Discussion}

This report describes 2 clinical cases in which strain elastography was used to assess palatal tumors in conjunction 

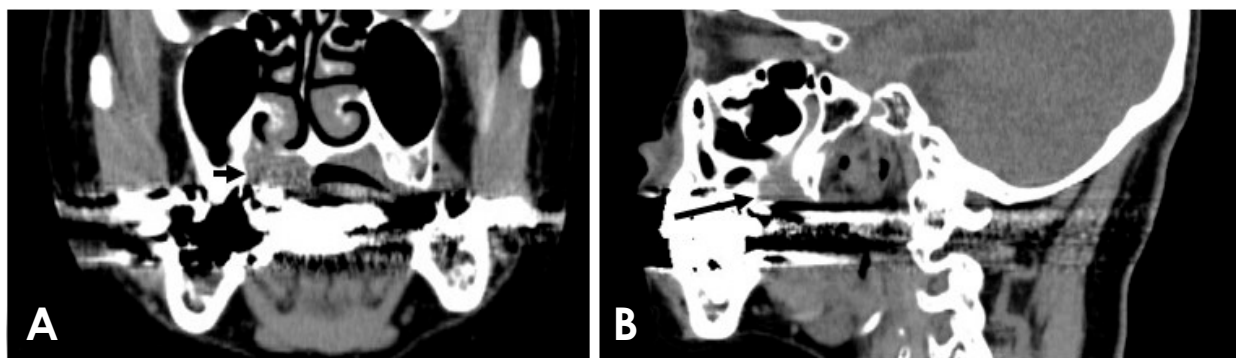

Fig. 6. Computed tomography (CT) of an adenoid cystic carcinoma of the palate in a 70 -year-old woman (arrows). A. Coronal soft-tissue algorithm CT image. B. Sagittal softtissue algorithm CT image. C. Coronal contrast-enhanced CT image. D. Sagittal contrast-enhanced CT image. E. Coronal bone-tissue algo-
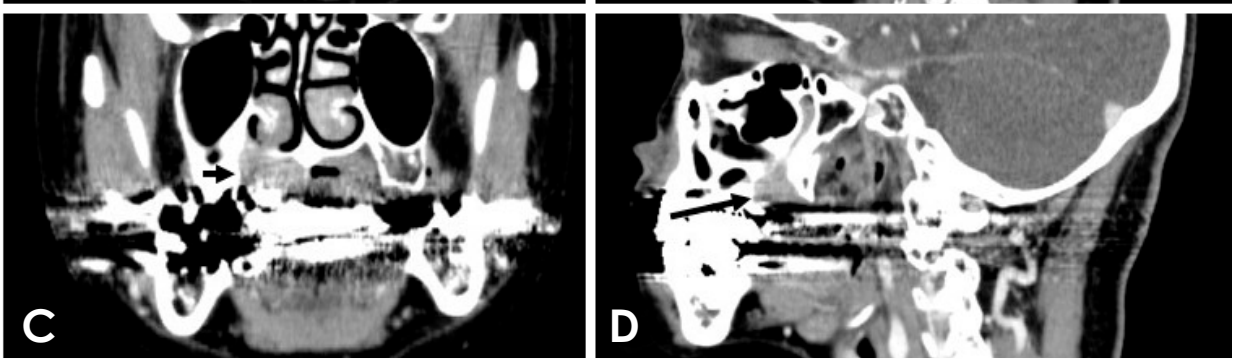
rithm CT image. F. Sagittal bone-tissue algorithm CT image.
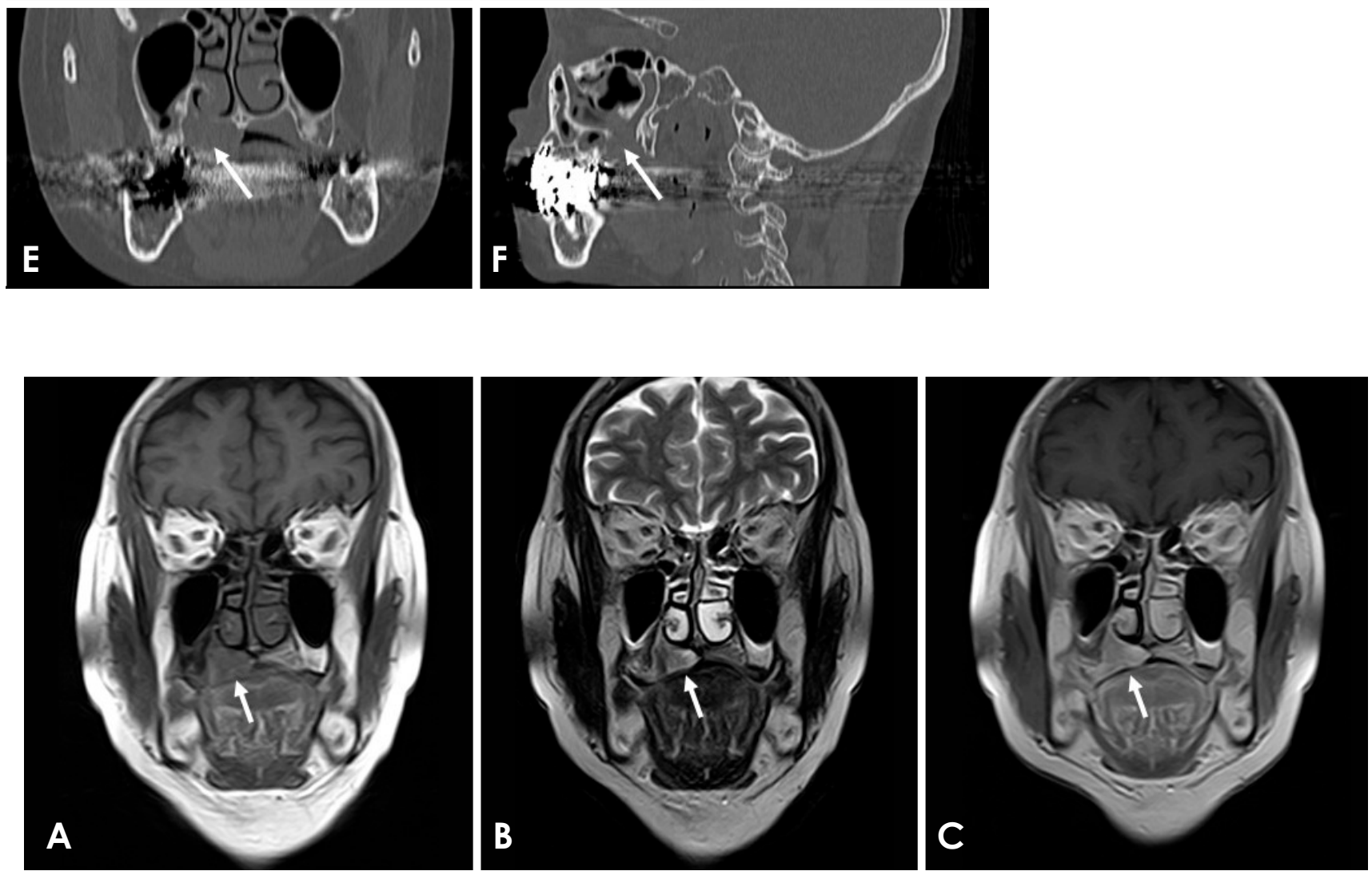

Fig. 7. Magnetic resonance imaging of an adenoid cystic carcinoma of the palate in a 70-year-old woman (arrows). A. Coronal T1-weighted image. B. Coronal T2-weighted image. C. Coronal post-contrast T1-weighted image.

with intraoral ultrasonography, CT, and MRI.

Regarding the imaging of palatal lesions, Kurabayashi et al. ${ }^{8}$ showed that on CT, malignant tumors of the minor salivary glands of the palate were very likely to display aggressive bone destruction, extension into the pterygopalatine fossa, and/or calcification. Ju et al. ${ }^{9}$ indicated that older patients with palatal tumors and intact mucosa should be considered for adenoid cystic carcinoma when CT shows relatively high palatine foramen enlargement. Yuan et al. ${ }^{10}$ showed that a combination of conventional MRI and apparent diffusion coefficient score could facilitate the differentiation of malignant from benign palatal lesions. Ishii et al. ${ }^{11}$ indicated that intraoral ultrasonography of palatal tumors could be used to determine the localization and condition 

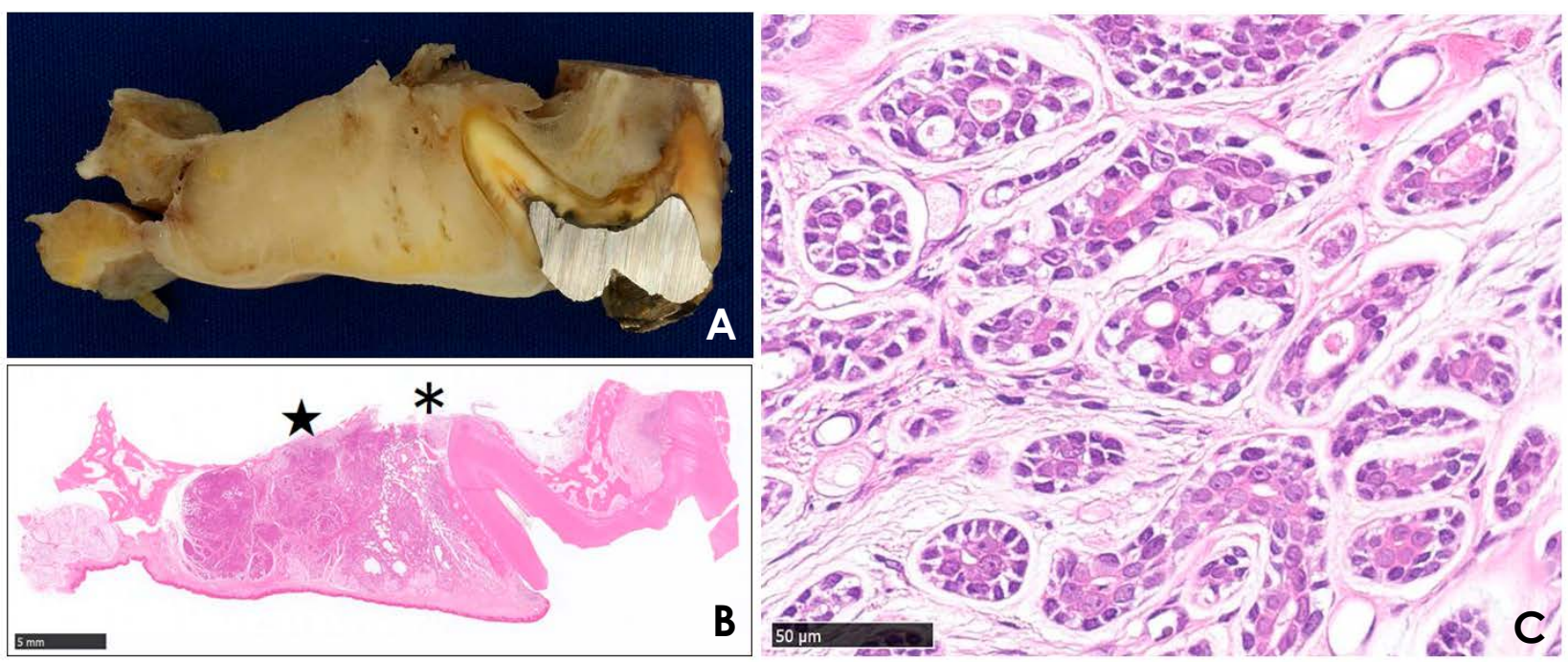

Fig. 8. Specimen and histopathological exam of an adenoid cystic carcinoma of the palate in a 70-year-old woman. In the coronal segment (A) of the right maxillary first molar (real view) and the low-magnification view (B) of the surgical specimen (H\&E stain $\times 1$; scale bar, 5 $\mathrm{mm})$, the tumor showed aggressive bone destruction and extension into the maxillary sinus $(*)$ and nasal cavity $(\star)$. C. Cribriform and tubular patterns were observed (H\&E stain $\times 40$; scale bar, $50 \mu \mathrm{m})$.

of tumors via close echogram analysis. The present report described 2 clinical cases (including both malignant and benign tumors) in which strain elastography was utilized to assess palatal tumors in conjunction with intraoral ultrasonography, CT, and MRI. We conclude that intraoral strain elastography may be useful for evaluating palatal lesions.

Inspection and palpation are frequently used for the clinical diagnosis of oral and maxillofacial lesions. Ogura et al. ${ }^{13-16}$ devised an instrument for measuring the consistency of oral cancer, quantitatively evaluated this consistency, and additionally evaluated the consistency of early invasive carcinoma of the oral cavity to enable the early detection of oral carcinoma. Along similar lines, we posit that using intraoral strain elastography in the assessment of palatal lesions may be helpful for the clinical diagnosis of early carcinoma of the palate.

The main limitation of our study is the possibility that patients may experience a few adverse effects from ultrasonography, including deep tissue heating, acoustic cavitation, and acoustic streaming. Ter Haar $^{17}$ described the existing laboratory and epidemiological evidence regarding the safety of diagnostic ultrasound.

In summary, we described 2 clinical cases in which strain elastography was used to assess palatal tumors along with intraoral ultrasonography, CT, and MRI. We conclude that intraoral strain elastography may be useful for evaluating palatal lesions.

\section{References}

1. Ogura I, Kaneda T, Sasaki Y, Sekiya K, Tokunaga S. Characteristic power Doppler sonographic images of tumorous and non-tumorous buccal space lesions. Dentomaxillofac Radiol 2013; 42: 20120460.

2. Ogura I, Nakahara K, Sasaki Y, Sue M, Oda T. Usefulness of shear wave elastography in the diagnosis of oral and maxillofacial diseases. Imaging Sci Dent 2018; 48: 161-5.

3. Sasaki Y, Ogura I. Shear wave elastography in differentiating between benign and malignant cervical lymph nodes in patients with oral carcinoma. Dentomaxillofac Radiol 2019; 48: 20180454.

4. Wakasugi-Sato N, Kodama M, Matsuo K, Yamamoto N, Oda M, Ishikawa A, et al. Advanced clinical usefulness of ultrasonography for diseases in oral and maxillofacial regions. Int $\mathbf{J}$ Dent 2010; 2010: 639382.

5. Sugawara C, Takahashi A, Kawano F, Kudo Y, Ishimaru N, Miyamoto Y. Intraoral ultrasonography of tongue mass lesions. Dentomaxillofac Radiol 2016; 45: 20150362.

6. Shingaki M, Nikkuni Y, Katsura K, Ikeda N, Maruyama S, Takagi $R$, et al. Clinical significance of intraoral strain elastography for diagnosing early stage tongue carcinoma: a preliminary study. Oral Radiol 2017; 33: 204-11.

7. Ogura I, Sasaki Y, Sue M, Oda T. Strain elastography of tongue carcinoma using intraoral ultrasonography: a preliminary study to characterize normal tissues and lesions. Imaging Sci Dent 2018; 48: 45-9.

8. Kurabayashi T, Ida M, Yoshino N, Sasaki T, Ishii J, Ueda M. Differential diagnosis of tumours of the minor salivary glands of the palate by computed tomography. Dentomaxillofac Radiol 1997; 26: 16-21.

9. Ju WT, Zhao TC, Liu Y, Tan YR, Dong MJ, Sun Q, et al. 
Computed tomographic features of adenoid cystic carcinoma in the palate. Cancer Imaging 2019; 19: 3.

10. Yuan Y, Tang W, Jiang M, Tao X. Palatal lesions: discriminative value of conventional MRI and diffusion weighted imaging. Br J Radiol 2016; 89: 20150911.

11. Ishii J, Nagasawa H, Wadamori T, Yamashiro M, Ishikawa $H$, Yamada $\mathrm{T}$, et al. Ultrasonography in the diagnosis of palatal tumors. Oral Surg Oral Med Oral Pathol Oral Radiol Endod 1999; 87: 39-43.

12. Ogura I, Oda T, Sue M, Sasaki Y, Hayama K. Comparison between squamous cell carcinoma and inflammatory diseases of the oral and maxillofacial region using gallium-67 scintigraphy with computed tomography and magnetic resonance imaging. Pol J Radiol 2018; 83: e452-8.

13. Ogura I, Amagasa T, Fujii E, Yoshimasu H. Quantitative evalu- ation of consistency in normal mucosa, leukoplakia, and squamous cell carcinoma of the gingiva. Oral Med Pathol 1997; 2: 69-73.

14. Ogura I, Amagasa T, Fujii E, Yoshimasu H. Quantitative evaluation of consistency of normal mucosa, leukoplakia and squamous cell carcinoma of the tongue. J Craniomaxillofac Surg 1998; 26: 107-11.

15. Ogura I, Amagasa T, Miyakura T, Fujii E, Sato M, Yoshimasu H. Quantitative evaluation of consistency of early invasive carcinoma of the tongue. Int J Clin Oncol 1999; 4: 220-3.

16. Ogura I, Amagasa T, Miyakura T. Correlation between tumor consistency and cervical metastasis in tongue carcinoma. Head Neck 2000; 22: 229-33.

17. Ter Haar G. Ultrasonic imaging: safety considerations. Interface Focus 2011; 1: 686-97. 